\title{
Licencia parental durante la educación y la práctica en medicina: 35 años de análisis bibliométrico
}

\section{Parental Leave during Medical Education and Practice: 35 years of Bibliometric Analysis}

\author{
Daisy Ximena Roa ${ }^{1}$ Silvia Riveros ${ }^{2}$ Maria Paula Saenz ${ }^{3}$ Joan Stefany Duque ${ }^{4(0)}$ \\ Catalina Sanchez $^{5}$ Catalina Solano ${ }^{6}$
}

${ }^{1}$ Uróloga, Cooperativa Especializada de servicios urológicos del

Address for correspondence Silvia Riveros García, M.D., Uróloga, Tolima, UROTOL

2 Uróloga, Compensar EPS, PAC, Colmedica MP

3 Uróloga, Clínica Universidad de la Sabana

${ }^{4}$ Uróloga, Hospital infantil universitario de San Jose. Instituto

Roosevelt

5 Uróloga, Clínica Nueva el Lago

6 Uróloga, Uroclin

Urol Colomb 2022;31(1):e12-e20. Compensar EPS, PAC, Colmedica MP, Dirección Carrera 16 \#127 B 43, torre 4, 202. Bogotá, Colombia (e-mail: sriverosg@hotmail.com).

\begin{abstract}
Resumen
Palabras clave

- asignación por maternidad

- médicos

- lactancia

- especialización

- bibliométrico
\end{abstract}

Objetivos La cantidad de mujeres en especialidades médico-quirúrgicas ha aumentado. Para nuestro conocimiento, no existen políticas que reglamenten las licencias parentales en nuestro país, que permitan a las mujeres que se desempeñan en esas especialidades vivir dicho periodo digna y equiparablemente con quienes han elegido otras carreras. Nuestro objetivo, es evaluar las publicaciones con respecto a la regulación y al apoyo a los procesos de maternidad y lactancia de los médicos.

Metodos Se realizó una revisión de la literatura, de los últimos 35 años en Medline a través de Fabumed, PubReminer y Scopus, utilizando los términos Mesh: "parental leave" y "physicians". Se incluyeron todas las publicaciones sobre licencia de maternidad en médicos, de 1984 a 2019. Se hizo un análisis bibliométrico descriptivo retrospectivo de dichos artículos y un análisis de mapeo bibliométrico utilizando el programa de software: VOSviewer. Se realizó un análisis estadístico descriptivo de los datos obtenidos.

Resultados Encontramos 182 publicaciones desde 1984 hasta 2019. Detectamos dos picos de publicación, en los años 1992 y 2018. El 39,6\% de las publicaciones está concentrado en 20 revistas, nueve con factor de impacto mayor a 3. La mayoría de las publicaciones (85\%) se encontraron en inglés y se originaron en 12 países.

Conclusiones Aún no hay suficientes publicaciones para generar consenso en cuanto a aspectos relacionados con la reglamentación de la licencia de maternidad y lactancia en cirujanas. La estandarización de políticas, optimiza el ejercicio de la especialidad al mejorar el grado de satisfacción de los involucrados. received

August 31, 2020 accepted after revision December 22, 2020
DOI https://doi.org/ $10.1055 / \mathrm{s}-0041-1724046$. ISSN 0120-789X. e ISSN 2027-0119. (c) 2022. Sociedad Colombiana de Urología. All rights reserved. This is an open access article published by Thieme under the terms of the Creative Commons Attribution-NonDerivative-NonCommercial-License, permitting copying and reproduction so long as the original work is given appropriate credit. Contents may not be used for commercial purposes, or adapted, remixed, transformed or built upon. (https://creativecommons.org/ licenses/by-nc-nd/4.0/)

Thieme Revinter Publicações Ltda., Rua do Matoso 170, Rio de Janeiro, RJ, CEP 20270-135, Brazil 
Abstract

\section{Keywords}

- parental leave

- physicians

- breastfeeding

- specialization

- bibliometrics
Objectives the number of women in a surgical medical specialty has increased over time. To our knowledge, there are no politics that rule parental leave in our country, with the goal of allowing women who work in these specialties to live through this period in a dignifying and comparable way as their equals who have made other career choices. The aim of this study is to assess the publications about regulation and support of maternity and breastfeeding for female doctors during their training and professional lives.

Methods A literature search using Mesh terms "parental leave" and "physicians", including articles published in the last 35 years, was performed through Medline, Fabumed, PubReminer and Scopus. Every publication regarding parental leave in medical doctors from 1984 to 2019 was included. Descriptive retrospective bibliometric analysis and bibliometric mapping analysis were performed of the above-mentioned articles using VOSviewer software. Finally, a statistical descriptive analysis was performed with the obtained data.

Results 184 publications from 1984 to 2019 were found. 2 Publication peaks were identified, in 1992 and 2018. 39.6\% of publications are concentrated in 20 journals, 9 of which have an Impact Factor greater than 3 . Most of the publications (85\%) were found in English language and originated in 12 countries.

Conclusions There are not enough publications to generate consensus related to the ruling of parental leave and breastfeeding in female surgeons. The standardization of politics for a specialty, optimizes its performance, as it improves the satisfaction degree of those involved.

\section{Introducción}

Con el paso del tiempo el número de mujeres en especializaciones médico-quirúrgicas ha aumentado por ejemplo, en urología, la incidencia de mujeres en residencia se ha incrementado desde un 9,4\% (1996) a un $21,3 \%$ (2006) y en la actualidad dicho porcentaje podría estar acercándose al 50\%. ${ }^{1}$

Con lo anterior, el embarazo y la planificación familiar (momento del parto o adopción) durante la especialización, se convierten en un tema cada vez más importante por aspectos como: los tiempos de licencia parental, las exigencias de los programas de educación, el mantenimiento de programas de especialización con altos estándares de calidad, las exigencias de la lactancia y crianza, el balance entre la vida familiar y el programa de educación médica continuada. En algunos casos se retrasa ese momento hasta terminar la especialización por las obligaciones, los horarios inflexibles y la preocupación sobre el ascenso en la carrera. ${ }^{2-4}$

Adicionalmente los problemas de fertilidad $y$ las complicaciones del embarazo y en la salud materno/fetal son más comunes entre cirujanos mujeres en comparación con la población en general, por prorrogar el momento de maternidad secundario a los factores anteriormente explicados. ${ }^{5,6}$

Dado que no hay políticas unánimes acerca de la licencia de maternidad, su duración, remuneración y planes de compensación, las redes sociales han surgido como un medio muy importante en la creación de canales de apoyo y soporte para las madres médicas en entrenamiento, ayudando a disminuir la insatisfacción y la frustración laboral. $^{7-10}$

Esas especialidades, en su génesis pensadas únicamente para hombres, tienen la misión de reestructurarse, teniendo en cuenta el ciclo vital de la mujer y su papel como madre, importante para la familia y para la sociedad.

Este análisis bibliométrico busca revisar y analizar la literatura disponible acerca de licencia de maternidad en mujeres médicas en los últimos 30 años.

\section{Métodos}

La base de datos MEDLINE fue consultada el 1 enero de 2020, a través de PubMed utilizando la estrategia de búsqueda: (Parental Leave, Physician) en todo periodo comprendido en el que se realizaron las publicaciones (1984 a 2019). Se incluyeron artículos originales de investigación, artículos de revista, ensayos, informes científicos y reseñas, con el fin de analizar toda la literatura médica indexada en relación con ese tema. Realizamos un análisis bibliométrico descriptivo de corte retrospectivo de la literatura médica disponible en la base de datos de MEDLINE en relación a LICENCIA DE MATERNIDAD EN MÉDICOS, existentes en la literatura. Las herramientas estadísticas FABUMED (un nuevo facilitador de búsquedas médicas), PubReminer y Scopus fueron utilizadas para la obtención de la información de las variables a analizar (número total de publicaciones y revistas, número y porcentaje de publicaciones por revista y país, y patrón de producción 
científica a lo largo del período estudiado, autores, artículos más citados). Mediante el software VOSviewer, utilizamos técnicas de agrupamiento ${ }^{11,12}$ que permiten analizar las interacciones de palabras clave de título y resumen de la literatura encontrada, con el fin de mapear el dominio del conocimiento científico y revelarle al lector una manera simple y rápida de ver los datos. Se adoptó el análisis de co-ocurrencia para investigar las áreas populares y direcciones de investigación y fue clave para monitorear desarrollos en áreas científicas.

Para la obtención del factor de impacto (FI), utilizamos el Journal Citation Reports 2017/2018 de las revistas encontradas para estimar la calidad de cada una de ellas. Todo el análisis fue realizado entre enero y abril del 2020. Posteriormente, analizamos los resultados de forma estadística y descriptiva y con base en esa información, elaboramos tablas y gráficas en Microsoft Office Excel 2017.

\section{Resultados}

Datos generales: Se recuperaron en total 182 publicaciones desde el año 1984 hasta 2019. Durante los 35 años de la revisión, hubo dos años con mayor número de publicaciones 1992 con 12 y 2019 con 16. Se dividió el tiempo en dos periodos por igual para determinar el crecimiento en las publicaciones. En el primer periodo (1984-2001) se publicaron $54(29,6 \%)$ y en el segundo periodo (20022019) se publicaron 117 (64,2\%) artículos, lo que demuestra un crecimiento del $34,6 \%$ en la última década. -Figura 1.
Revistas: Los 182 artículos fueron publicados en 111 revistas - Figura 2. El 37,9\% (69) de las publicaciones se dieron en las 20 revistas demostradas en la -Tabla 1, así como el número de artículos publicados en cada una de ellas y el FI para el año 2018 según el Journal Citation Reports. La revista con mayor número de publicaciones fue: Acad Med con ocho artículos, seguida de J Am Med Womens Assoc (1972) con siete y JAMA con seis. Solamente 1\% (2) de las revisiones fueron publicadas en revistas de urología: J Urol y BJU Int. En la - Tabla 1 vemos como cuatro del top 20 de revistas, presentaron un factor de impacto mayor a 8, la revista con mayor FI fue Annals of Internal Medicine con 19,31. El 85\% de los artículos se encontraban en inglés.

Países: La producción total de publicaciones se originó en 28 países. El país con más número de publicaciones fue Estados Unidos 96 (52,7\%), seguido de Alemania con 20 (10,9\%), Canadá 15 (8,2\%) y Reino Unido 9 (4,94\%). Ningún país de América Latina contó con publicaciones - Figura 3.

Instituciones: De las diez instituciones principales, dos pertenecen a Alemania (Universitätsklinikum Ulm y Universitätsklinikum Heidelberg) y las otras a Estados Unidos. La Institución con mayor número de artículos publicados es la Universidad de Harvard (11). En la - Figura 4 aparecen los porcentajes teniendo en cuenta las instituciones principales y no el total de los artículos.

Autores y tipo de publicaciones: El promedio de artículos publicados por autor fue dos. Los autores con mayor número de publicaciones fueron dos mujeres, cada una con cuatro: Jagsi, R de la University of Michigan Medical School, Ann Arbor, Estados Unidos, tiene 9701 citaciones y un h-índex

20

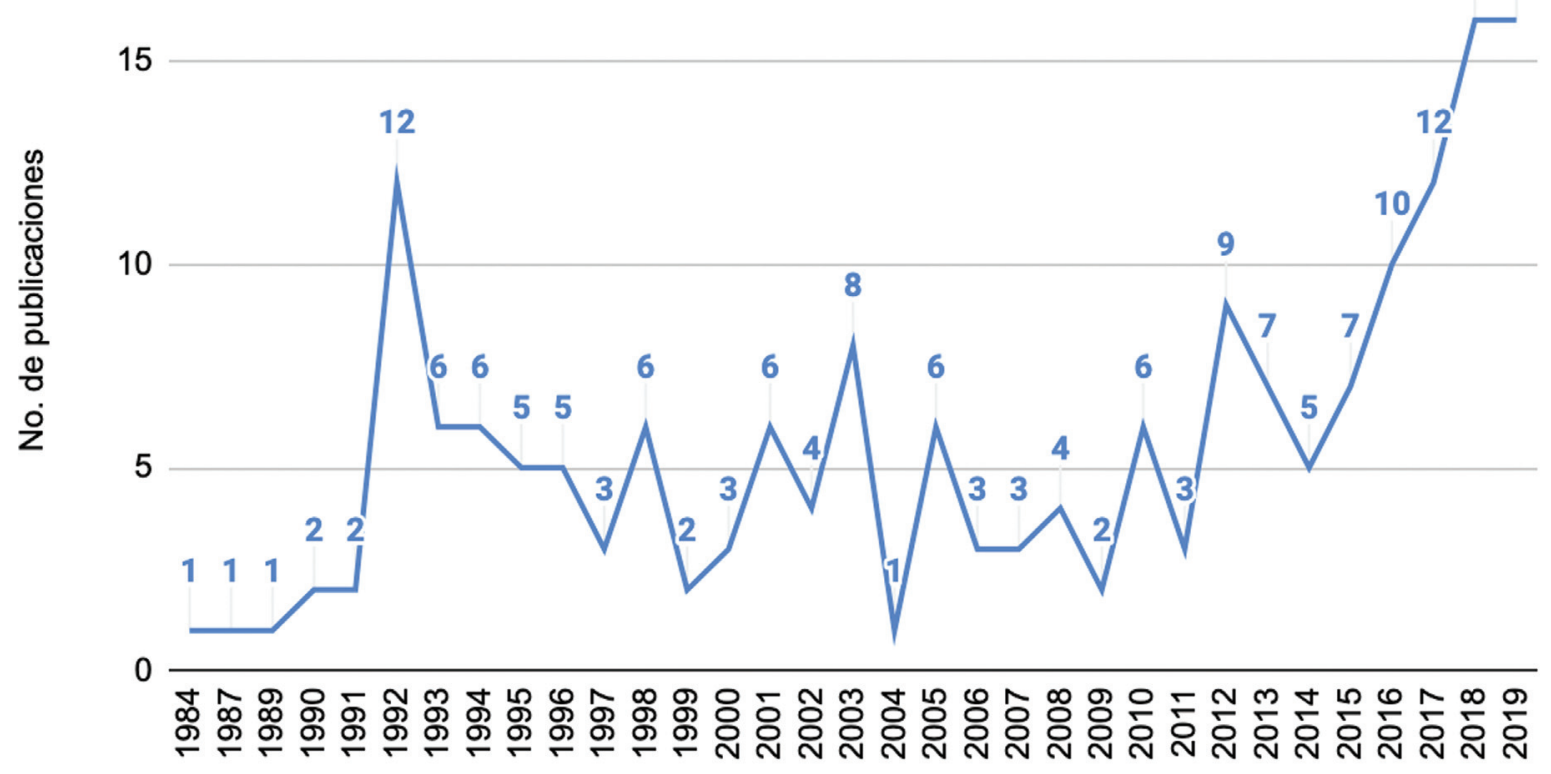

Año

Fig. 1 Crecimiento de publicaciones globales en las últimas tres décadas acerca de licencia de maternidad en médicos. 


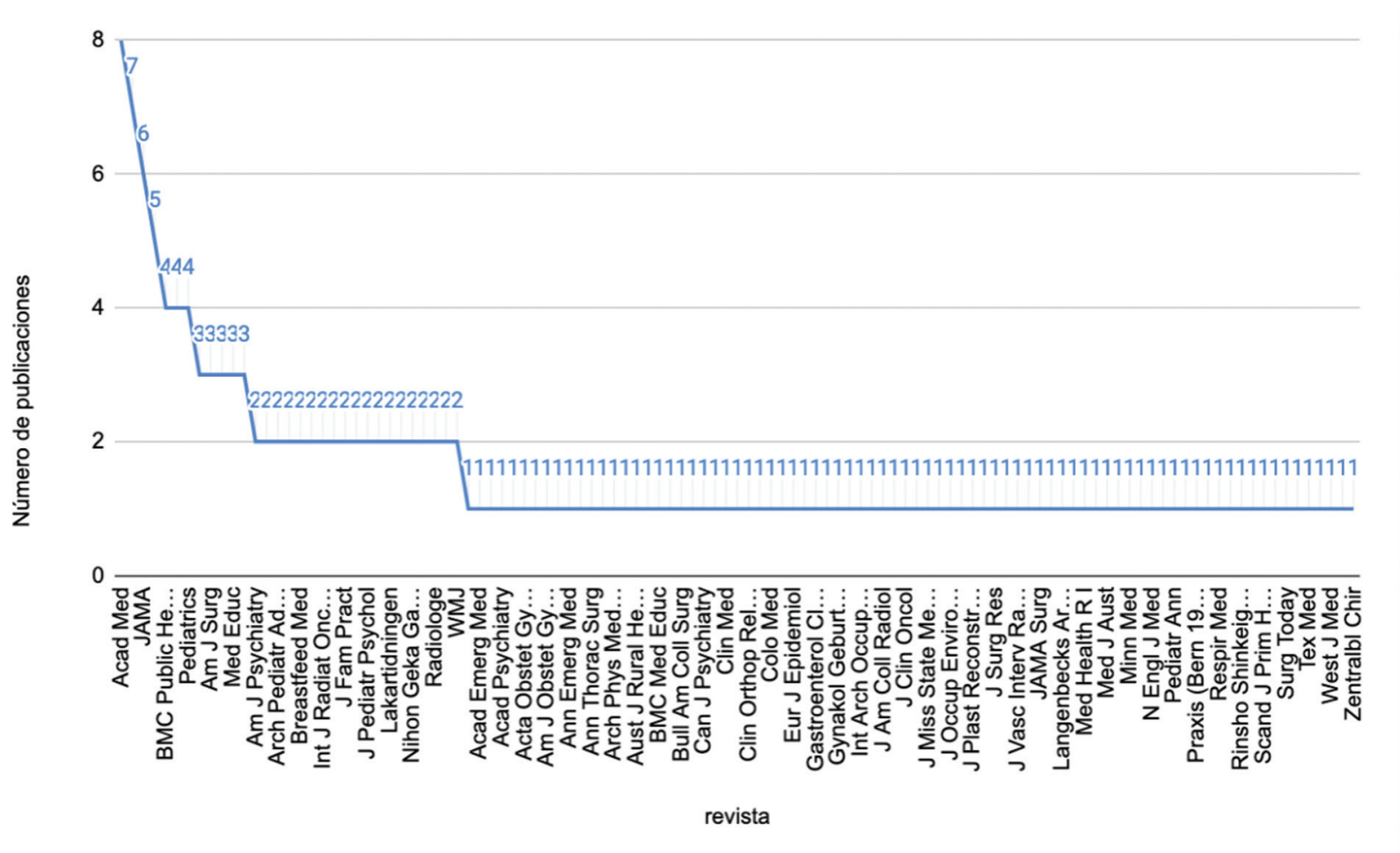

Fig. 2 Distribución porcentual de revistas con publicación sobre licencia de maternidad en médicos, durante el período 1984-2019.

Tabla 1 Listado de revistas con publicación de artículos sobre licencia de maternidad en médicos, durante el período 1984 a 2019 , su factor de impacto para el año 2017-2018, el número de publicaciones, el idioma. (- no se reportó Fl para este periodo)

\begin{tabular}{|l|l|l|l|l|}
\hline Revista & Artículos & $\begin{array}{l}\text { Factor de impacto } \\
2017-2018\end{array}$ & Idioma & País \\
\hline Acad Med & 8 & 4,93 & Inglés & Estados Unidos \\
\hline J Am Med Womens Assoc (1972) & 7 & - & Inglés & Estados Unidos \\
\hline JAMA & 6 & 10,66 & Inglés & Estados Unidos \\
\hline GMS Z Med Ausbild & 5 & - & Inglés & Alemania \\
\hline BMC Public Health & 4 & 2,56 & Inglés & Inglaterra \\
\hline CMAJ (Canadian medical association journal) & 4 & 6,93 & Multi-lenguaje & Canadá \\
\hline Pediatrics & 4 & 5,40 & Inglés & Estados Unidos \\
\hline Am J Gastroenterol & 3 & 10,24 & Inglés & Estados Unidos \\
\hline Am J Surg & 3 & 2,20 & Inglés & Estados Unidos \\
\hline Fam Med & 3 & 1,08 & Inglés & Estados Unidos \\
\hline Med Educ & 3 & 4,61 & Inglés & Inglaterra \\
\hline Plast Reconstr Surg & 3 & 3,94 & Inglés & Inglaterra \\
\hline Am J Psychiatry & 3 & 13,65 & Inglés & Estados Unidos \\
\hline Ann Intern Med & 2 & 19,31 & Inglés & Estados Unidos \\
\hline Arch Pediatr Adolesc Med & 2 & 2,29 & Inglés & Estados Unidos \\
\hline Arch Surg & 2 & - & Inglés & Estados Unidos \\
\hline Breastfeed Med & 2 & 1,52 & Inglés & Estados Unidos \\
\hline Can Fam Physician & 2 & 2,18 & Multi-lenguaje & Canadá \\
\hline Int J Radiat Oncol Biol Phys & 2 & - & Inglés & Estados Unidos \\
\hline J Am Med Womens Assoc & 2 & - & Inglés & Estados Unidos \\
\hline
\end{tabular}




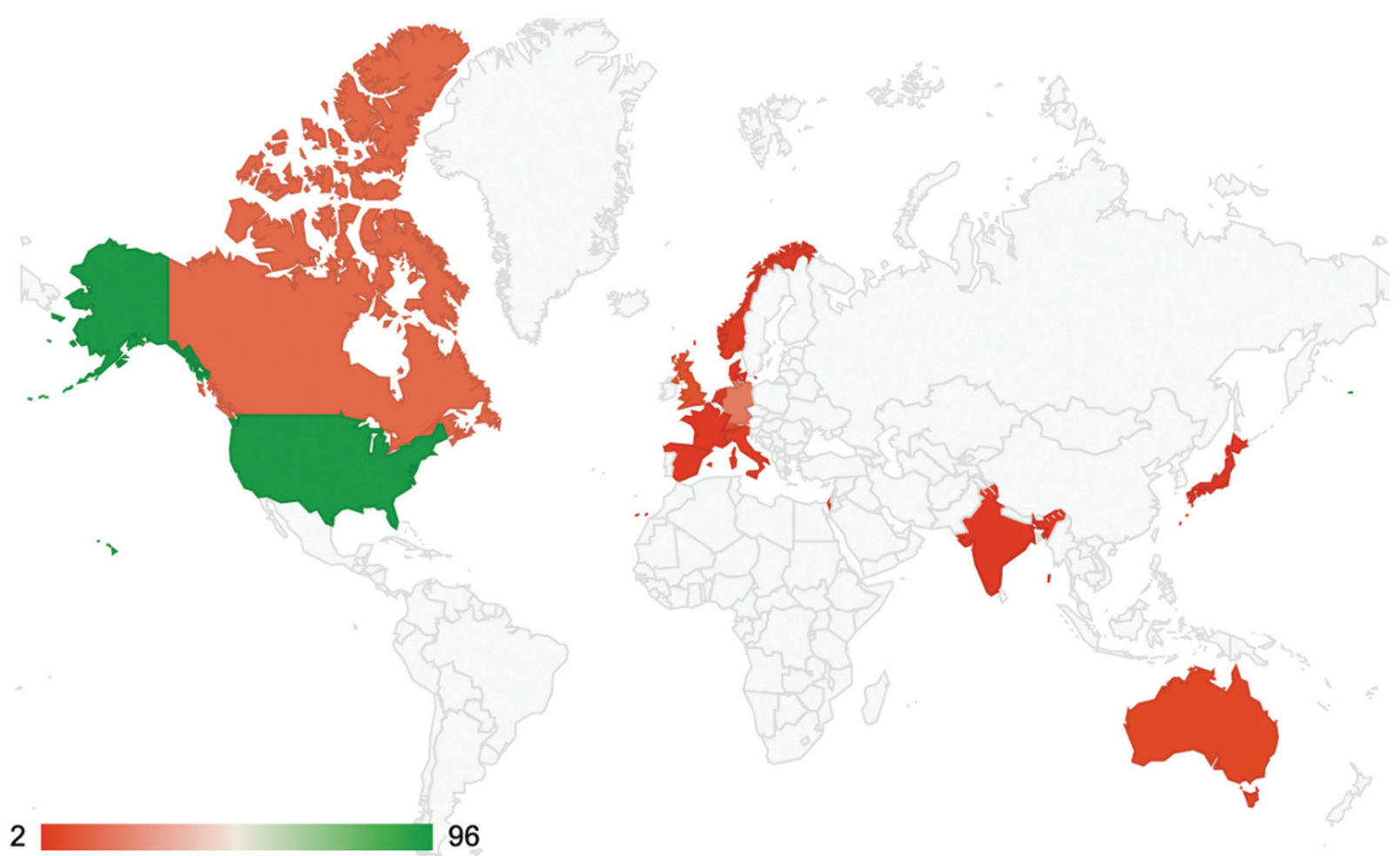

Fig. 3 Países participantes en las publicaciones de licencia de maternidad en médicos en el periodo desde 1984 a 2019.

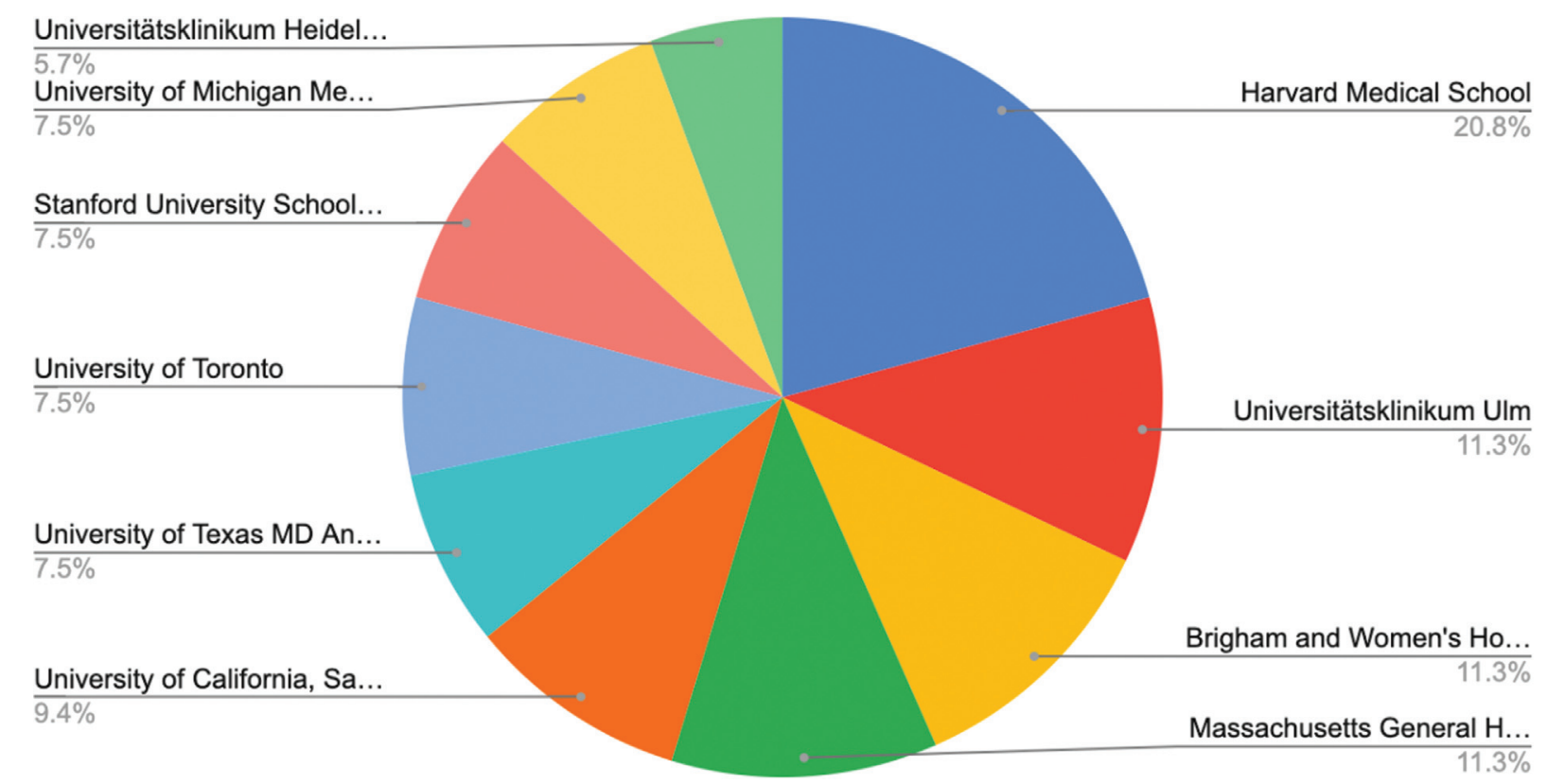

Fig. 4 Principales instituciones participantes en las publicaciones.

de 54 y Lent, B de la Western University, London, con un total de 1343 citaciones y h-index de 17. El tipo de publicación más frecuente fue en los artículos originales, en un 69,2\%. - Figura 5.

Artículos más citados: Cuando analizamos los diez artículos más citados, se encuentra en primer lugar Pregnancy during residency: A literature review, publicado en 2003 por Susan J Finch, con un total de 75 citaciones, publicada en ACADEMIC MEDICINE (FI 4,93). Otro punto a tener en cuenta es que en casi todas las especialidades se ha publicado acerca del tema y su relación con la satisfacción laboral. La revista con mayor FI $(20,58)$ en las publicaciones es: en JOURNAL OF THE AMERICAN COLLEGE OF CARDIOLOGY seguido por JAMA surgery. El $90 \%$ son documentos tipo artículo. - Tabla 2.

Análisis de co-ocurrencia de palabras claves: Esas correlaciones le permiten al lector hacer un mapeo rápido acerca de los tópicos predominantes en la literatura 


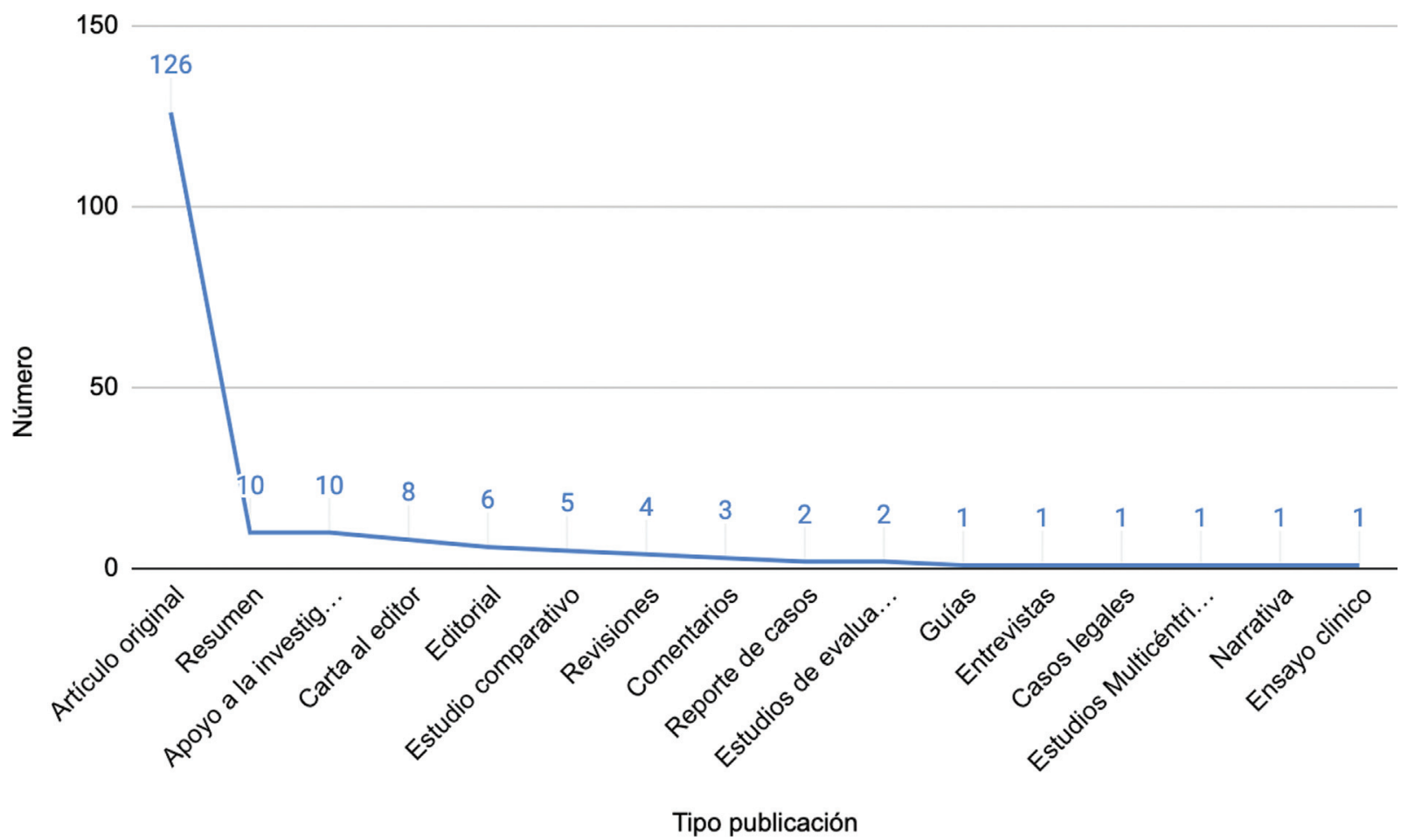

Fig. 5 Tipo de publicaciones acerca de licencia de maternidad en médicos.

relacionada con "parental leave, physicians". En la - Tabla 3 encontramos las palabras clave con mayor número de co-ocurrencia, las cuales son: "humans" (176), "female" (154), "parental leave" (134) y "physicians women" (103). Al mapear y analizar dichas palabras clave en gráficos de red con un mínimo de 5 repeticiones, se obtienen 81 de ellas divididas en cinco grupos. A continuación, se describirán los tres grupos principales: Grupo 1 (rojo) hace referencia a las mujeres médicas madres, adultos jóvenes o adultos en edad media con: complicaciones en el embarazo, licencia por enfermedad, retorno laboral, horas laborales. Grupo 2 (verde) indica el vínculo entre: licencia de maternidad en mujeres médicas, estudiantes y cirujanas y los derechos de la mujer en sitios de trabajo, exigencia de horarios en sitios de trabajo, cambio laboral. Grupo 3 (azul) reúne nuevamente la licencia de maternidad y mujeres médicas, con el rol de madre, cuidado de sus hijos, la práctica familiarQ4. (- Figura 6).

\section{Discusión}

El embarazo y la maternidad durante la residencia o la práctica médica pueden convertirse en un conflicto multifacético, estrechamente relacionado con factores laborales y políticas de la licencia de maternidad, que afectan la lactancia y pueden generar problemas de salud materna y familiar. A pesar de un posible aumento en la carga laboral impuesta a las colegas durante esa licencia, es importante destacar que el embarazo y la paternidad son una valiosa experiencia de aprendizaje, aportando a la humanización de un departamento médico. Lo anterior queda plasmado en la estructura conceptual de esta investigación, en el mapa de co-ocurrencia de palabras clave.
Sin embargo, en lo que atañe a la licencia de maternidad en mujeres médicas, en la literatura mundial, por el momento no se han encontrado iniciativas en las que se caracterice la actividad científica, evaluando tendencias en autorías, las citas y la puntuación de impacto sobre los artículos. Por lo que este es el primer análisis bibliométrico sobre publicaciones relacionadas con ese aspecto.

Al evaluar los indicadores de actividad se considera un tema poco detallado, en el que no se tiene claro el campo específico de publicación (182 artículos fueron publicados en 111 revistas de diferentes campos de estudio). Con un comportamiento en el tiempo oscilatorio, un promedio de 1 artículo escrito por autor y escasa complejidad de las investigaciones $(65 \%$ de la literatura encontrada fue artículos originales). Destacándose en el impacto científico individual a Jagsi, R, con un h-índex relevante de 54 y Lent, B, con h-index de 17; mientras que de forma institucional sobresale Harvard Medical School. Lo anterior unido al número de autores, artículos y citaciones encontradas, demuestran su baja popularidad.

A través de la vida media y dinámica de citación que es claramente prolongada (los dos artículos más citados tienen más de 17 años); se demuestra que es un campo que evoluciona lentamente. Sin embargo, la ventana de citación de los artículos más antiguos es mucho más amplia que los publicados en los últimos años y teniendo en cuenta un crecimiento en la publicación del $34 \%$ en la última década; se considera que el impacto de los últimos estudios sobre el tema aún está por verse.

En los últimos años, la fuerza laboral médica femenina ha aumentado a nivel mundial, aunque más en el entorno clínico que en el quirúrgico; siendo el año 2017, la primera vez en la 
Tabla 2 Los diez artículos con mayor número de publicaciones, con su autor, revista de publicaciones, palabras clave por autor y tipo documento

\begin{tabular}{|c|c|c|c|c|c|c|}
\hline Autores & Titulo & Año & Revista & Citas & Palabras clave & $\begin{array}{l}\text { Tipo } \\
\text { documento }\end{array}$ \\
\hline Finch S.J. & $\begin{array}{l}\text { Pregnancy during residen- } \\
\text { cy: A literature review }\end{array}$ & 2003 & Academic Medicine & 75 & & Artículo \\
\hline $\begin{array}{l}\text { Young-Shumate L., } \\
\text { Kramer T., Beresin E. }\end{array}$ & $\begin{array}{l}\text { Pregnancy during graduate } \\
\text { medical training }\end{array}$ & 1993 & Academic Medicine & 30 & & Artículo \\
\hline $\begin{array}{l}\text { Merchant S.J., } \\
\text { Hameed S.M., Melck } \\
\text { A.L. }\end{array}$ & $\begin{array}{l}\text { Pregnancy among residents } \\
\text { enrolled in general surgery: } \\
\text { A nationwide survey of atti- } \\
\text { tudes and experiences }\end{array}$ & 2013 & $\begin{array}{l}\text { American Journal of } \\
\text { Surgery }\end{array}$ & 29 & $\begin{array}{l}\text { Maternity; } \\
\text { Policy; } \\
\text { Pregnancy; } \\
\text { Residency; } \\
\text { Surgery }\end{array}$ & Artículo \\
\hline $\begin{array}{l}\text { Lerner L.B., Baltrushes } \\
\text { R.J., Stolzmann K.L., } \\
\text { Garshick E. }\end{array}$ & $\begin{array}{l}\text { Satisfaction of Women } \\
\text { Urologists With Maternity } \\
\text { Leave and Childbirth Timing }\end{array}$ & 2010 & Journal of Urology & 29 & $\begin{array}{l}\text { breast feeding; } \\
\text { parental leave; } \\
\text { parturition; } \\
\text { urology; women }\end{array}$ & Artículo \\
\hline $\begin{array}{l}\text { Cole S., Arnold M., } \\
\text { Sanderson A., Cupp C. }\end{array}$ & $\begin{array}{l}\text { Pregnancy during otolaryn- } \\
\text { gology residency: Experi- } \\
\text { ence and recommendations }\end{array}$ & 2009 & American Surgeon & 28 & & Artículo \\
\hline $\begin{array}{l}\text { Holliday E.B., Ahmed } \\
\text { A.A., Jagsi R., Stentz } \\
\text { N.C., Woodward W. } \\
\text { A., Fuller C }\end{array}$ & $\begin{array}{l}\text { Pregnancy and parenthood } \\
\text { in radiation oncology, views } \\
\text { and experiences survey } \\
\text { (PROVES): Results of a } \\
\text { blinded prospective trainee } \\
\text { parenting and career devel- } \\
\text { opment assessment }\end{array}$ & 2015 & $\begin{array}{l}\text { International Journal } \\
\text { of Radiation Oncology } \\
\text { Biology Physics }\end{array}$ & 21 & & Artículo \\
\hline $\begin{array}{l}\text { Sarma A.A., Nkonde- } \\
\text { Price C., Gulati M., } \\
\text { Duvernoy C.S., Lewis } \\
\text { S.J., Wood M.J. }\end{array}$ & $\begin{array}{l}\text { Cardiovascular Medicine } \\
\text { and Society: The Pregnant } \\
\text { Cardiologist }\end{array}$ & 2017 & $\begin{array}{l}\text { Journal of the Ameri- } \\
\text { can College of } \\
\text { Cardiology }\end{array}$ & 18 & $\begin{array}{l}\text { pregnancy; } \\
\text { training; } \\
\text { women in } \\
\text { cardiology }\end{array}$ & Revisión \\
\hline $\begin{array}{l}\text { Scully R.E., Davids J.S., } \\
\text { Melnitchouk N. }\end{array}$ & $\begin{array}{l}\text { Impact of Procedural Spe- } \\
\text { cialty on Maternity Leave } \\
\text { and Career Satisfaction } \\
\text { among Female Physicians }\end{array}$ & 2017 & Annals of Surgery & 16 & $\begin{array}{l}\text { career choice; } \\
\text { career satisfaction; } \\
\text { financial burden; } \\
\text { maternity leave; } \\
\text { physicians; women }\end{array}$ & Artículo \\
\hline $\begin{array}{l}\text { Manaster B.J., Hulen } \\
\text { R. }\end{array}$ & $\begin{array}{l}\text { Pregnancy and maternity } \\
\text { policies in radiology resi- } \\
\text { dencies: The } 1993 \text { survey of } \\
\text { the american association for } \\
\text { women radiologists }\end{array}$ & 1995 & Academic Radiology & 15 & & Artículo \\
\hline $\begin{array}{l}\text { Rangel E.L., Lyu H., } \\
\text { Haider A.H., Castillo- } \\
\text { Angeles M., Doherty } \\
\text { G.M., Smink D.S. }\end{array}$ & $\begin{array}{l}\text { Factors Associated with } \\
\text { Residency and Career Dis- } \\
\text { satisfaction in Childbearing } \\
\text { Surgical Residents }\end{array}$ & 2018 & JAMA Surgery & 10 & & Artículo \\
\hline
\end{tabular}

historia en que el número de mujeres que se inscribieron en las escuelas de medicina de EE. UU. excedió el número de hombres. ${ }^{13,14}$ Pero esos cambios no se han visto reflejados en la inclusión laboral junto a cambios en la licencia de maternidad, avances en las políticas, incluso solo se encontró $1 \%$ de publicación en revistas urológicas, siendo una especialidad con un número creciente de mujeres en los últimos años.

Llama la atención, que en nuestros resultados se presentaron dos picos con mayor número de publicaciones, 1992 con 12/174 y 2018 con 16/174. Eso se explica porque en 1991 la asociación médica americana adoptó nuevas políticas en maternidad que impulsaban los programas de educación médica a delinear políticas, pólizas con beneficios y ajustes salariales con respecto a la licencia de maternidad, logrando que durase como mínimo 6 semanas. ${ }^{15,16}$ Mientras que la producción del 2018 es el resultado de que, en el año anterior, The American board of surgery (ABS), reformuló sus políticas para lograr la flexibilidad en los programas de residencia, incluyendo el tiempo de licencia de paternidad en el entrenamiento médico. ${ }^{17}$ Las creaciones de políticas estandarizadas generan impactos importantes y positivos en la práctica y en la actividad científica.

Entonces, por asociarse directamente a la evolución de esas políticas en la medicina de cada país y ser un tema no específico para su divulgación; logra ser más susceptible de publicación en revistas especializadas que en multidisciplinarias. Del top 20 de revistas, solo 4 presentan un FI mayor a 8, de ellas solo una es multidisciplinaria (JAMA) y las otras tres de especialidades no quirúrgicas. Eso podría responder la tendencia de FI bajos, dado que existen diferencias entre la intensidad de investigación en 
Tabla 3 Palabras clave respecto a "parental leave, physicinas"

\begin{tabular}{|l|l|}
\hline Palabras clave & Ocurrencias \\
\hline Humans & 176 \\
\hline Female & 154 \\
\hline Parental leave & 134 \\
\hline Physicians, women & 103 \\
\hline Male & 85 \\
\hline Adult & 63 \\
\hline Pregnancy & 72 \\
\hline Intership and residency & 50 \\
\hline United states & 56 \\
\hline Surveys and questionnaires & 47 \\
\hline Child & 32 \\
\hline Middle aged & 27 \\
\hline Career choice & 28 \\
\hline Attitude of health personnel & 27 \\
\hline Child, preschool & 19 \\
\hline Physicians & 23 \\
\hline Infant & 17 \\
\hline Child care & 16 \\
\hline Workload & 14 \\
\hline Organizational policy & 17 \\
\hline Workforce & 14 \\
\hline Time factors & 19 \\
\hline & \\
\hline
\end{tabular}

los diferentes campos de estudios (especialidades), lo que hace que varíe el FI de especialidad en especialidad; además de que es frecuente encontrar FI más altos en revistas multidisciplinarias.

A pesar de los beneficios demostrados de la protección de la lactancia y la maternidad durante los programas de medicina, solo en los países del primer mundo hay alguna forma de auxilios o lineamientos, algo que va muy de la mano con la producción literaria, donde el 22,9\% de los artículos se originan en Estados unidos, Inglaterra, Canadá y Alemania. No se encontraron estudios en Latinoamérica, eso es un reflejo claro de pobres políticas defensoras y probablemente escasa preparación formal en investigación en nuestro medio; por lo que los estudios bibliométricos son más valorados en países científicamente desarrollados. Igualmente en nuestros países, la producción literaria durante los años de residencia, está dirigida a favorecer el conocimiento y no se centra en mejorar la calidad ni las condiciones de vida de los especialistas en formación. Sumado a que los profesionales suelen preferir lo asistencial frente a la investigación y publicación.

Sin embargo, el análisis de co-ocurrencia de palabras clave en los estudios demuestra el creciente interés científico en aspectos más humanos de las mujeres médicas como lo es el embarazo y la licencia de maternidad. Las madres médicas necesitan lograr un balance entre el trabajo y el rol familiar, que tiene implicaciones especiales en el núcleo familiar y finalmente, en la sociedad. Por lo que también toman importancia términos como la elección de carrera, derechos de la mujer en sitios de trabajo, tolerancia al horario de trabajo,

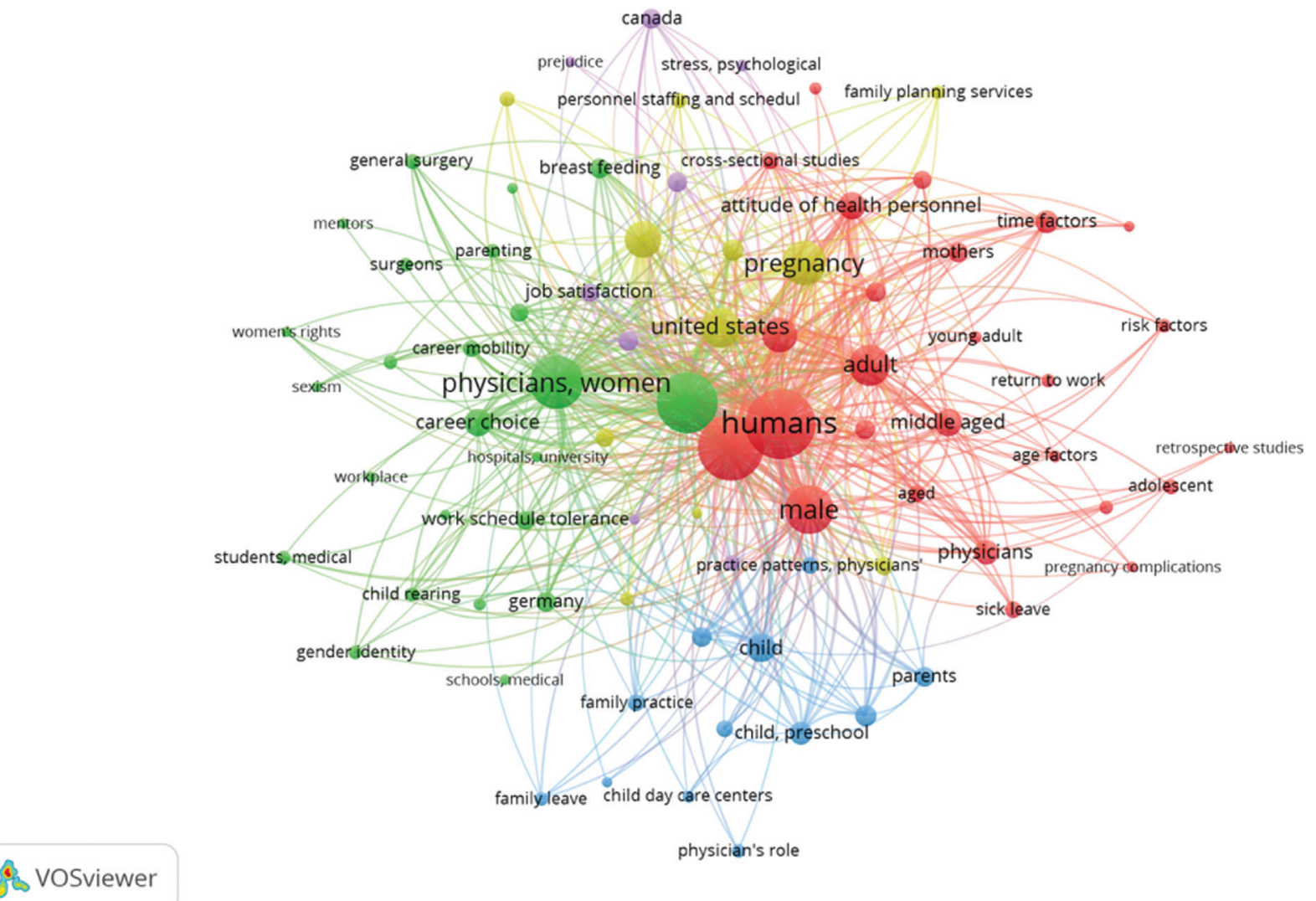

Fig. 6 Mapa de co-ocurrencia de palabras clave relacionadas con "parental leave physicians. 
lactancia, licencias por enfermedad, satisfacción laboral entre otros campos de estudio. No obstante, el mapa demuestra que esa comunidad científica tiene un hábito de colaboración bajo, lo que es esencial para mejorar esa inequidad política y científica en lo que atañe a la licencia de maternidad en el ámbito médico.

Dentro de las limitantes, se encontró que las publicaciones en PubMed son principalmente en inglés, desfavoreciendo a países que publican en revistas en un idioma diferente, así mismo solo se registra la dirección del primer autor en los artículos de revistas. Por esa razón, se tuvieron en cuenta todos los tipos de artículos indexados, incluyendo artículos de revisión, artículos originales de investigación, reseñas, ensayos y otros informes científicos. La estrategia de búsqueda utilizada para la recolección bibliografía ha sido usada por diferentes autores, por lo que nuestros resultados serán comparables con futuros manuscritos. A pesar de las limitaciones mencionadas, este estudio es un instrumento útil para investigadores, convirtiéndose en el punto de partida para definir las necesidades de futuras líneas de investigación, fortaleciendo la producción científica en urología.

\section{Conclusión}

La literatura es insuficiente para generar un consenso que determine el tiempo adecuado y las condiciones óptimas de la licencia de maternidad, en mujeres en entrenamiento de especialidades médico-quirúrgicas o en especialistas en esos campos y no refleja la situación de la población médica en general.

La creación de políticas estandarizadas aumenta el grado de satisfacción con las especialidades y con el ejercer de las mismas, haciéndolas aún más necesarias por las mujeres médicas que aspiran a la formación como especialistas. La solución para que esa licencia sea remunerada con una duración y garantías adecuadas sigue siendo un desafío apremiante.

Esperamos que el impacto del crecimiento en el número de publicaciones que se ha visto en la última década se vea reflejado en la creación de políticas a ese nivel y poder contribuir de forma activa en su elaboración.

Conflicto de intereses

Los autores declaran no tener ningún conflicto de intereses.

\section{Referencias}

1 Sayres M, Wyshak G, Denterlein G, Apfel R, Shore E, Federman D. Pregnancy during residency. N Engl J Med 1986;314(07):418-423

2 Tamburrino MB, Evans CL, Campbell NB, Franco KN, Jurs SG, Pentz JE. Physician pregnancy: male and female colleagues' attitudes. J Am Med Womens Assoc (1972) 1992;47(03):82-84

3 Bickel J. Women in medical education. A status report. N Engl J Med 1988;319(24):1579-1584

4 Franco K, Evans CL, Best AP, Zrull JP, Pizza GA. Conflicts associated with physicians' pregnancies. Am J Psychiatry 1983;140(07): 902-904

5 Turner PL, Lumpkins K, Gabre J, Lin MJ, Liu X, Terrin M. Pregnancy among women surgeons: trends over time. Arch Surg 2012;147 (05):474-479

6 Hutchinson AM, Anderson NS III, Gochnour GL, Stewart C. Pregnancy and childbirth during family medicine residency training. Fam Med 2011;43(03):160-165

7 Rose SH, Burkle CM, Elliott BA, Koenig LF. The impact of parental leave on extending training and entering the board certification examination process: a specialty-based comparison. Mayo Clin Proc 2006;81(11):1449-1453

8 Cole S, Arnold M, Sanderson A, Cupp C. Pregnancy during otolaryngology residency: experience and recommendations. Am Surg 2009;75(05):411-415

9 Association of American Medical Colleges. Table 27: Total graduates by US medical school and sex, 2010-2014. https:// www.aamc.org/download/321532/data/factstable27-2.pdf. Accessed September 30, 2015

10 Smith C, Galante JM, Pierce JL, Scherer LA. The surgical residency baby boom: changing patterns of childbearing during residency over a 30-year span. J Grad Med Educ 2013;5(04):625-629

11 Van Eck NJ, Waltman L, Dekker R, Van Den Berg J. A comparison of two techniques for bibliometric mapping: multidimensional scaling and VOS. J Accessed 13 Sep 2018

12 Waltman L, van Eck NJ, Noyons ECM. A unified approach to mapping and clustering of bibliometric networks. 2010http:// arxiv.org/abs/1006.1032

13 American Association of Medical Colleges Graduate Medical Education Track. (2006 Data) and American Medical Association (1996 Data). American Association of Medical Colleges. Accessed 2009 at www.aamc.org

14 American Association of Medical Colleges. The State of Women in Academic Medicine: The Pipeline and Pathways to Leadership, 2015-2016. AAMC GME

15 Brower BA, Jennings MM, Butterworth ML, Crawford ME. Women in Podiatry and Medicine. Clin Podiatr Med Surg 2019;36(04): 707-716

16 Burkhart J. AMA-RPS instrumental in achieving new maternity leave policy. JAMA 1991;265(13):1756

17 Varda BK Glover M. Specialty board leave policies for resident physicians requesting parental leave. JAMA 2018;320(22): 2374-2377 